\title{
ON THE ZERO SET OF SEMI-INVARIANTS FOR QUIVERS
}

\author{
BY CHRISTINE RIEDTMANN AND GRZEGORZ ZWARA
}

ABSTRACT. - Let $\mathbf{d}$ be a prehomogeneous dimension vector for a finite quiver $Q$. We show that the set of common zeros of all semi-invariants of positive degree for the variety of representations of $Q$ with dimension vector $N \cdot \mathbf{d}$ under the product of the general linear groups at all vertices is irreducible and a complete intersection for large natural numbers $N$.

(C) 2003 Elsevier SAS

RÉSUMÉ. - Soient $Q$ un carquois fini et $\mathbf{d}$ un vecteur de dimension pour $Q$ tels que la variété des représentations de $Q$ de dimension $\mathbf{d}$ contienne une orbite dense sous l'action du groupe $\mathrm{Gl}(\mathbf{d})$ des changements de base en chaque sommet. Nous montrons que l'ensemble des zéros des semi-invariants de degré positif sous $\operatorname{Gl}(N \cdot \mathbf{d})$ sur la variété des représentations de dimension $N \cdot \mathbf{d}$ est irréductible et une intersection complète pourvu que $N$ soit suffisamment grand.

(C) 2003 Elsevier SAS

\section{Introduction}

Let $k$ be an algebraically closed field, and let $Q=\left(Q_{0}, Q_{1}, t, h\right)$ be a finite quiver, i.e. a finite set $Q_{0}=\{1, \ldots, n\}$ of vertices and a finite set $Q_{1}$ of arrows $\alpha: t \alpha \rightarrow h \alpha$, where $t \alpha$ and $h \alpha$ denote the tail and the head of $\alpha$, respectively.

A representation of $Q$ over $k$ is a collection $\left(X(i) ; i \in Q_{0}\right)$ of finite dimensional $k$-vector spaces together with a collection $\left(X(\alpha): X(t \alpha) \rightarrow X(h \alpha) ; \alpha \in Q_{1}\right)$ of $k$-linear maps. A morphism $f: X \rightarrow Y$ between two representations is a collection $(f(i): X(i) \rightarrow Y(i))$ of $k$-linear maps such that

$$
f(h \alpha) \circ X(\alpha)=Y(\alpha) \circ f(t \alpha) \quad \text { for all } \alpha \in Q_{1} .
$$

The dimension vector of a representation $X$ of $Q$ is the vector

$$
\operatorname{dim} X=(\operatorname{dim} X(1), \ldots, \operatorname{dim} X(n)) \in \mathbb{N}^{Q_{0}} .
$$

We denote the category of representations of $Q$ by $\operatorname{rep}(Q)$, and for any vector $\mathbf{d}=\left(d_{1}, \ldots, d_{n}\right) \in \mathbb{N}^{Q_{0}}$

$$
\operatorname{rep}(Q, \mathbf{d})=\prod_{\alpha \in Q_{1}} \operatorname{Mat}\left(d_{h \alpha} \times d_{t \alpha}, k\right)
$$

is the vector space of representations $X$ of $Q$ with $X(i)=k^{d_{i}}, i \in Q_{0}$. The group

$$
\mathrm{Gl}(\mathbf{d})=\prod_{i=1}^{n} \mathrm{Gl}\left(d_{i}, k\right)
$$


acts on $\operatorname{rep}(Q, \mathbf{d})$ by

$$
\left(\left(g_{1}, \ldots, g_{n}\right) \star X\right)(\alpha)=g_{h \alpha} \cdot X(\alpha) \cdot g_{t \alpha}^{-1} .
$$

Note that the $\operatorname{Gl}(\mathbf{d})$-orbit of $X$ consists of the representations $Y$ in $\operatorname{rep}(Q, \mathbf{d})$ which are isomorphic to $X$.

We call $\mathbf{d}$ a prehomogeneous dimension vector if $\operatorname{rep}(Q, \mathbf{d})$ contains an open orbit $\operatorname{Gl}(\mathbf{d}) \star T$. Such a representation $T$ is characterized by $\operatorname{Ext}_{Q}^{1}(T, T)=0$ [6]. If $Q$ admits only finitely many indecomposable representations, or equivalently if the underlying graph of $\bar{Q}$ is a disjoint union of Dynkin diagrams $\mathbb{A}, \mathbb{D}$ or $\mathbb{E}[2]$, every vector $\mathbf{d}$ is prehomogeneous. Indeed, any representation is a direct sum of indecomposables in an essentially unique way by the theorem of KrullSchmidt, and therefore $\operatorname{rep}(Q, \mathbf{d})$ contains finitely many orbits, one of which must be open.

Let $\mathbf{d}$ be prehomogeneous, and let $f_{1}, \ldots, f_{s} \in k[\operatorname{rep}(Q, \mathbf{d})]$ be the irreducible monic polynomials whose zeros $Z\left(f_{1}\right), \ldots, Z\left(f_{s}\right)$ are the irreducible components of codimension 1 of $\operatorname{rep}(Q, \mathbf{d}) \backslash \mathrm{Gl}(\mathbf{d}) \star T$, where $\mathrm{Gl}(\mathbf{d}) \star T$ is the open orbit. It is easy to see that

$$
g \cdot f_{i}=\chi_{i}(g) \cdot f_{i}
$$

for $g \in \mathrm{Gl}(\mathbf{d})$, where $\chi_{i}: \mathrm{Gl}(\mathbf{d}) \rightarrow k^{*}$ is a character. A regular function with this property is called a semi-invariant. By [8], any semi-invariant is a scalar multiple of a monomial in $f_{1}, \ldots, f_{s}$, and $f_{1}, \ldots, f_{s}$ are algebraically independent. We denote by

$$
\mathcal{Z}_{Q, \mathbf{d}}=\left\{X \in \operatorname{rep}(Q, \mathbf{d}) ; f_{i}(X)=0, i=1, \ldots, s\right\}
$$

the set of common zeros of all semi-invariants of positive degree. Obviously we have $\operatorname{codim} \mathcal{Z}_{Q, \mathbf{d}} \leqslant s$, and equality means that $\mathcal{Z}_{Q, \mathbf{d}}$ is a complete intersection.

Our first main result is as follows.

THEOREM 1.1. - Let $T_{1}, \ldots, T_{r}$ be pairwise non-isomorphic indecomposable representations in $\operatorname{rep}(Q)$ such that $\operatorname{Ext}_{Q}^{1}\left(T_{i}, T_{j}\right)=0$ for any $i, j \leqslant r$. Then there is a positive integer $N$ such that $\mathcal{Z}_{Q, \mathrm{~d}}$ is a complete intersection and an irreducible variety for any dimension vector $\mathbf{d}=\sum_{i=1}^{r} \lambda_{i} \cdot \operatorname{dim} T_{i}$ with $\lambda_{i} \geqslant N, i \leqslant r$.

As an immediate consequence we derive the following fact.

COROLlaRY 1.2. - Let $\mathbf{d}$ be a prehomogeneous dimension vector in $\mathbb{N}^{Q_{0}}$. Then there is a positive integer $N$ such that $\mathcal{Z}_{c \cdot \mathbf{d}}$ is a complete intersection and an irreducible variety for any $c \geqslant N$.

We will prove in a forthcoming paper that we may choose $N=2$ if $\bar{Q}$ is a disjoint union of Dynkin diagrams and $N=3$ if $\bar{Q}$ is a disjoint union of Dynkin diagrams and extended Dynkin diagrams.

In order to put our results into the context of invariant theory, we recall a few definitions. We assume that $k$ is the field of complex numbers. Let $G$ be a reductive algebraic group acting regularly on a finite dimensional vector space $V$. By Hilbert's theorem, the ring $k[V]^{G}$ of $G$-invariant polynomials on $V$ is a finitely generated algebra and thus is the algebra of polynomial functions on a variety $V / / G$. The inclusion of $k[V]^{G}$ into $k[V]$ gives rise to a regular surjective map $\pi: V \rightarrow V / / G$ which is constant on $G$-orbits, the so-called categorical quotient of $V$ by $G$ [3]. As $G$ is completely reducible, the $G$-module $k[V]$ can be decomposed uniquely as a direct sum

$$
k[V]=\bigoplus_{\lambda} M_{\lambda} \otimes_{k} V_{\lambda}
$$


where $M_{\lambda}$ ranges over a set of representatives of the irreducible $G$-modules and $V_{\lambda}$ is just a vector space, possibly infinite dimensional, which records the multiplicity with which $M_{\lambda}$ arises in $k[V]$. As the action of $G$ on $k[V]$ commutes with multiplication by $G$-invariants, each $V_{\lambda}$ can be viewed as a $k[V]^{G}$-module. In fact, (1.1) is a decomposition as $G$ - $k[V]^{G}$-bimodules; the group $G$ acts only on $M_{\lambda}$ and $k[V]^{G}$ only on $V_{\lambda}$. A covariant of weight $\lambda$ is a $G$-linear map

$$
\varphi: k[V] \rightarrow M_{\lambda}
$$

or equivalently a linear form on $V_{\lambda}$. The pair $(V, G)$ is called:

- coregular if $V / / G$ has no singularities,

- equidimensional if the fiber $\pi^{-1}(\pi(0))$ has the same dimension as the quotient $V / / G$,

- cofree if $k[V]$ is free as a $k[V]^{G}$-module, or equivalently the module $V_{\lambda}^{*}$ of covariants is free over $k[V]^{G}$ for all $\lambda$.

An equidimensional coregular pair $(V, G)$ is automatically cofree ([5], [12] §17). G. Schwarz classified all coregular and cofree representations of connected simple algebraic groups [10,11]. In [4] P. Littelmann classified all cofree irreducible representations of semisimple groups.

Let us consider $V=\operatorname{rep}(Q, \mathbf{d})$ for a prehomogeneous $\mathbf{d}$ as a representation of the subgroup $\mathrm{Sl}(\mathbf{d})=\prod_{i=1}^{n} \operatorname{Sl}\left(d_{i}\right)$ of $\mathrm{Gl}(\mathbf{d})$. For each arrow $\alpha$, the set

$$
V_{\alpha}=\{X \in V ; X(\beta)=0 \forall \beta \neq \alpha\}
$$

is an irreducible subrepresentation of $V$, and $V$ is the direct sum $V=\bigoplus_{\alpha \in Q_{1}} V_{\alpha}$. The ring $k[V]^{\mathrm{Sl}(\mathbf{d})}$ of $\mathrm{Sl}(\mathbf{d})$-invariants is generated by the semi-invariants $f_{1}, \ldots, f_{s}$. So the categorical quotient $V / / \mathrm{Sl}(\mathbf{d})$ is an $s$-dimensional affine space and $(V, \mathrm{Sl}(\mathbf{d}))$ is coregular. It is cofree in the cases for which our main results mentioned above hold. Surprisingly, the situation is better for big multiples of a given dimension vector. It can be bad otherwise, as the following example illustrates: For

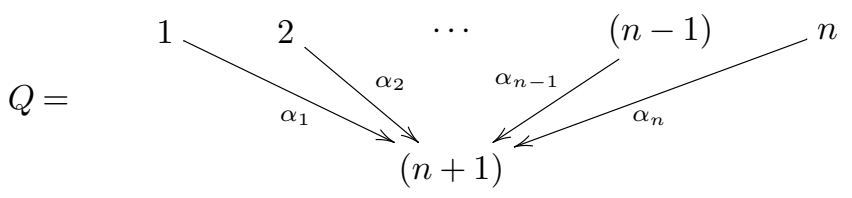

the dimension vector $\mathbf{d}=(1, \ldots, 1, n-1)$ is prehomogeneous. Indeed, the open orbit in $\operatorname{rep}(Q, \mathbf{d})$ consists of those representations $X$ for which none of the $(n-1) \times(n-1)$-minors $f_{1}, \ldots, f_{n}$ of the $(n-1) \times n$-matrix $\left[X\left(\alpha_{1}\right), \ldots, X\left(\alpha_{n}\right)\right]$ vanishes. On the other hand, the set $\mathcal{Z}_{Q, \mathbf{d}}$ of common zeros of $f_{1}, \ldots, f_{n}$ is the set of $(n-1) \times n$-matrices of rank less than $n-1$ and thus has codimension 2. In [1], Chang and Weyman examine quivers $Q$ with underlying graph $\mathbb{A}_{n}$ and arbitrary dimension vectors. They find that $(\operatorname{rep}(Q, \mathbf{d}), \operatorname{Sl}(\mathbf{d}))$ is always equidimensional, but the set $\pi^{-1}(\pi(0))=\mathcal{Z}_{Q, \mathbf{d}}$ is reducible in general.

\section{Notations and preliminaries}

We first recall Schofield's construction of semi-invariants from [9]. The Euler form is the $\mathbb{Z}$-bilinear form on $\mathbb{Z}^{Q_{0}}$ defined by

$$
\langle\mathbf{d}, \mathbf{e}\rangle=\sum_{i \in Q_{0}} d_{i} e_{i}-\sum_{\alpha \in Q_{1}} d_{t \alpha} e_{h \alpha}
$$


For $\mathbf{d}, \mathbf{e} \in \mathbb{N}^{Q_{0}}, X \in \operatorname{rep}(Q, \mathbf{d}), Y \in \operatorname{rep}(Q, \mathbf{e})$ we consider the linear map

$$
\mathcal{F}_{X, Y}: \bigoplus_{i \in Q_{0}} \operatorname{Hom}_{k}\left(k^{d_{i}}, k^{e_{i}}\right) \rightarrow \bigoplus_{\alpha \in Q_{1}} \operatorname{Hom}_{k}\left(k^{d_{t \alpha}}, k^{e_{h \alpha}}\right)
$$

which sends $\left(g_{i} ; i \in Q_{0}\right)$ to $\left(h_{\alpha} ; \alpha \in Q_{1}\right)$ with $h_{\alpha}=g_{h \alpha} \cdot X(\alpha)-Y(\alpha) \cdot g_{t \alpha}$. Note that

$$
\operatorname{ker} \mathcal{F}_{X, Y}=\operatorname{Hom}_{Q}(X, Y) \text { and } \operatorname{coker} \mathcal{F}_{X, Y} \simeq \operatorname{Ext}_{Q}^{1}(X, Y) .
$$

This implies that

$$
\langle\operatorname{dim} X, \operatorname{dim} Y\rangle=[X, Y]-{ }^{1}[X, Y]
$$

where we set

$$
[X, Y]=\operatorname{dim}_{k} \operatorname{Hom}_{Q}(X, Y), \quad{ }^{1}[X, Y]=\operatorname{dim}_{k} \operatorname{Ext}_{Q}^{1}(X, Y) .
$$

If we assume that $\langle\mathbf{d}, \mathbf{e}\rangle=0$, the linear map $\mathcal{F}_{X, Y}$ will be represented by a square matrix $M_{X, Y}$ (with respect to some bases), and the determinant $\operatorname{det} M_{X, Y}$ is a $\mathrm{Gl}(\mathbf{d}) \times \mathrm{Gl}(\mathbf{e})$-semi-invariant on $\operatorname{rep}(Q, \mathbf{d}) \times \operatorname{rep}(Q, \mathbf{e})$. It might vanish, however.

For a representation $U$ of $Q$, the right perpendicular category $U^{\perp}$ and the left perpendicular category ${ }^{\perp} U$ are the full subcategories of $\operatorname{rep}(Q)$ whose objects $Y$ satisfy

$$
[U, Y]={ }^{1}[U, Y]=0 \text { and }[Y, U]={ }^{1}[Y, U]=0,
$$

respectively. As ${ }^{1}[X, Y]=[Y, \tau X]$ for any two representations $X$ and $Y$ of $Q$, where $\tau$ is the Auslander-Reiten translation (see [7] for relevant definitions), we have $U^{\perp}={ }^{\perp}(\tau U)$.

Now assume that $T_{1}, \ldots, T_{r}$ are pairwise non-isomorphic with ${ }^{1}\left[T_{i}, T_{j}\right]=0, i, j=1, \ldots, r$ and such that $T=\bigoplus_{i=1}^{r} T_{i}^{\lambda_{i}}$ is sincere, i.e., $T(e) \neq 0$ for all $e \in Q_{0}$. Then the category $T^{\perp}$ is equivalent to the category of representations of a quiver $Q^{\perp}$ having $(n-r)$ vertices. Choose $Y \in T^{\perp}, Y \neq 0$, and set $\mathbf{d}=\operatorname{dim} T, \mathbf{e}=\operatorname{dim} Y$. Observe that

$$
\langle\mathbf{d}, \mathbf{e}\rangle=[T, Y]-{ }^{1}[T, Y]=0,
$$

the dimension of $\bigoplus_{i \in Q_{0}} \operatorname{Hom}_{k}\left(k^{d_{i}}, k^{e_{i}}\right)$ is positive and $M_{T, Y}$ is invertible. Thus the $\mathrm{Gl}(\mathbf{d})$ semi-invariant $f_{Y}=\operatorname{det} M_{X, Y}$ is non-trivial on $\operatorname{rep}(Q, \mathbf{d})$. It is easy to see that $f_{Y}=f_{Y^{\prime}} \cdot f_{Y^{\prime \prime}}$ for an exact sequence $0 \rightarrow Y^{\prime} \rightarrow Y \rightarrow Y^{\prime \prime} \rightarrow 0$ in $T^{\perp}$. If the simple objects of $T^{\perp}$ are $S_{1}, \ldots, S_{n-r}$, the semi-invariants $f_{S_{1}}, \ldots, f_{S_{n-r}}$ are algebraically independent and they generate the algebra of $\mathrm{Sl}(\mathbf{d})$-invariants. As a consequence we have

$$
\begin{aligned}
\mathcal{Z}_{Q, \mathbf{d}} & =\left\{X \in \operatorname{rep}(Q, \mathbf{d}) ;\left[X, S_{j}\right] \neq 0, j=1, \ldots, n-r\right\} \\
& =\left\{X \in \operatorname{rep}(Q, \mathbf{d}) ;[X, Y] \neq 0 \text { for all } Y \in T^{\perp}, Y \neq 0\right\} .
\end{aligned}
$$

We will keep the following assumptions and notations throughout the paper: $T$ is a sincere representation of $Q$ with ${ }^{1}[T, T]=0$ and $\operatorname{dim} T=\mathbf{d}$. We can always make $T$ sincere by considering the full subquiver which supports $T$ instead of $Q$. Observe that $Q$ does not contain oriented cycles. If the decomposition of $T$ as a direct sum of pairwise non-isomorphic indecomposables is

$$
T=\bigoplus_{i=1}^{r} T_{i}^{\lambda_{i}}, \quad \lambda_{i} \geqslant 1,
$$


we set $\lambda=\min \left\{\lambda_{i}: i=1, \ldots, r\right\}$. Note that $\mathcal{Z}_{Q, \mathbf{d}}$ is defined by $n-r$ polynomial equations. In order to prove it is a complete intersection it suffices to show

$$
\operatorname{codim} \mathcal{Z}_{Q, \mathbf{d}} \geqslant n-r
$$

All varieties we consider will be quasi-projective over $k$, and we will look at codimensions for constructible subsets of affine space only.

\section{Proof of Theorem 1.1}

Our strategy is to first get rid of the set $\left\{X \in \mathcal{Z}_{Q, \mathbf{d}} ;{ }^{1}[T, X] \neq 0\right\}$ by showing its codimension is big. In fact, it is for this we need our assumption on the multiplicities $\lambda_{i}$.

The following lemma will be used several times in our article.

Lemma 3.1. - Let $\mathbf{d}^{\prime \prime} \in \mathbb{N}^{Q_{0}} \backslash\{0\}$ be such that $\mathbf{d}^{\prime \prime} \leqslant \mathbf{d}$, i.e., $\mathbf{d}^{\prime}=\mathbf{d}-\mathbf{d}^{\prime \prime} \in \mathbb{N}^{Q_{0}}$, and let $V=V_{1} \oplus \cdots \oplus V_{b}$ belong to $\operatorname{rep}\left(Q, \mathbf{d}^{\prime \prime}\right)$, where $V_{1}, \ldots, V_{b}$ are indecomposable. Then the set

$$
\mathcal{A}_{V}=\{X \in \operatorname{rep}(Q, \mathbf{d}) ; \exists \text { epimorphism } X \rightarrow V\}
$$

is constructible, irreducible, and $\operatorname{codim} \mathcal{A}_{V} \geqslant b-\left\langle\mathbf{d}, \mathbf{d}^{\prime \prime}\right\rangle$.

Proof. - Consider the subvariety

$$
\mathcal{C}=\left\{\left(X, g=\left[\begin{array}{l}
g^{\prime} \\
g^{\prime \prime}
\end{array}\right]\right) ; g^{\prime \prime} \in \operatorname{Hom}_{Q}(X, V)\right\}
$$

of $\operatorname{rep}(Q, \mathbf{d}) \times \operatorname{Gl}(\mathbf{d})$. Note that $g^{\prime \prime}$ is an epimorphism as $g$ is invertible. This leads to the surjective regular map $\pi: \mathcal{C} \rightarrow \mathcal{A}_{V}$ given by the first projection. We see that the set $\mathcal{A}_{V}$ is constructible and that

$$
\operatorname{dim} \pi^{-1}(X)=[X, V]+\sum_{i \in Q_{0}} d_{i}^{\prime} d_{i} \geqslant b+\sum_{i \in Q_{0}} d_{i}^{\prime} d_{i}
$$

for $X \in \mathcal{A}_{V}$, since there exists an epimorphism $X \rightarrow V_{1} \oplus \cdots \oplus V_{b}$.

On the other hand, sending $(X, g)$ to $(g \star X, g)$ we obtain an isomorphism from $\mathcal{C}$ to the subvariety $\mathcal{D}$ of $\operatorname{rep}(Q, \mathbf{d}) \times \operatorname{Gl}(\mathbf{d})$ consisting of all pairs $(Y, g)$ for which $Y(\alpha)$ is in the block form

$$
Y(\alpha)=\left[\begin{array}{cc}
* & * \\
0 & V(\alpha)
\end{array}\right], \quad \alpha \in Q_{1} .
$$

As $\mathcal{D}$ is just the product of an affine space of dimension $\sum_{\alpha \in Q_{1}} d_{h \alpha}^{\prime} d_{t \alpha}$ with $\mathrm{Gl}(\mathbf{d})$, we conclude that $\mathcal{A}_{V}$ is irreducible and that

$$
\sum_{\alpha \in Q_{1}} d_{h \alpha}^{\prime} d_{t \alpha}+\operatorname{dim} \operatorname{Gl}(\mathbf{d})-\operatorname{dim} \mathcal{A}_{V}=\operatorname{dim} \mathcal{C}-\operatorname{dim} \mathcal{A}_{V} \geqslant b+\sum_{i \in Q_{0}} d_{i}^{\prime} d_{i}
$$

Our estimate follows from an easy computation.

COROLLARY 3.2. - Keeping the notations of the preceding lemma, we assume moreover that $V$ is a subrepresentation of $\tau T$. Then we have

$$
\operatorname{codim} \mathcal{A}_{V} \geqslant 1+\lambda
$$


Proof. - It suffices to show $-\left\langle\mathbf{d}, \mathbf{d}^{\prime \prime}\right\rangle \geqslant \lambda_{i}$ for some $i$. Observe that

$$
[T, V] \leqslant[T, \tau T]={ }^{1}[T, T]=0 \quad \text { and } \quad{ }^{1}[T, V]=[V, \tau T]>0 .
$$

Consequently, we have ${ }^{1}\left[T_{i}, V\right] \geqslant 1$ for some $i$ and thus

$$
-\left\langle\mathbf{d}, \mathbf{d}^{\prime \prime}\right\rangle=-[T, V]+{ }^{1}[T, V]={ }^{1}[T, V] \geqslant{ }^{1}\left[T_{i}^{\lambda_{i}}, V\right] \geqslant \lambda_{i} .
$$

For any $U \in \operatorname{rep}(Q)$, we denote by $\mathcal{X}_{U}$ the set

$$
\mathcal{X}_{U}=\{X \in \operatorname{rep}(Q, \mathbf{d}) ;[X, U] \neq 0\} .
$$

LEMMA 3.3. - Let $U$ be a non-zero subrepresentation of $\tau T$. Then we have

$$
\operatorname{codim} \mathcal{X}_{U} \geqslant \lambda+1-\eta(\operatorname{dim} U),
$$

where $\eta(\mathbf{e})=\sum_{i \in Q_{0}}\left[e_{i}^{2} / 4\right]$, for any $\mathbf{e} \in \mathbb{N}^{Q_{0}}$, and $[q]$ denotes the largest integer not exceeding $q$ for $q \in \mathbb{Q}$.

Proof. - We want to exploit that for any non-zero homomorphism $\varphi: X \rightarrow U$ from $X \in \operatorname{rep}(Q, \mathbf{d})$ to $U, X$ belongs to $\mathcal{A}_{V}$ for the representation $V=\varphi(X)$, which is a quotient of $X$ as well as a subrepresentation of $\tau T$. Set $\mathbf{e}=\operatorname{dim} U$, and choose $\mathbf{f} \in \mathbb{N}^{Q_{0}}$ with $\mathbf{f} \leqslant \mathbf{e}, \mathbf{d}$. Consider the closed subvariety $\mathcal{L}_{\mathbf{f}}$ of $\prod_{i \in Q_{0}} \operatorname{Grass}\left(k^{e_{i}}, f_{i}\right)$ consisting of sequences $\left(V_{i}\right)_{i \in Q_{0}}$ such that $U(\alpha)\left(V_{t \alpha}\right) \subseteq V_{h \alpha}, \alpha \in Q_{1}$. In other words, $\mathcal{L}_{\mathbf{f}}$ is the variety of all subrepresentations $V$ of $U$ with dimension vector $\mathbf{f}$. Note that $\operatorname{dim} \mathcal{L}_{\mathbf{f}} \leqslant \eta(\mathbf{e})$ since $\operatorname{dim} \operatorname{Grass}\left(k^{e}, f\right)=(e-f) f \leqslant\left[e^{2} / 4\right]$. The subset $\mathcal{F}_{\mathbf{f}}$ of $\mathcal{L}_{\mathbf{f}} \times \operatorname{rep}(Q, \mathbf{d})$ consisting of pairs $(V, X)$ such that there is an epimorphism from $X$ onto $V$ is constructible. Indeed, the affine subvariety

$$
\mathcal{H}=\left\{\left(\varphi=\left(\varphi_{i}\right), X\right) ; \varphi \in \operatorname{Hom}_{Q}(X, U)\right\} \subseteq \prod_{i \in Q_{0}} \operatorname{Hom}_{k}\left(k^{d_{i}}, k^{e_{i}}\right) \times \operatorname{rep}(Q, \mathbf{d})
$$

is the disjoint union $\coprod_{\mathbf{f} \leqslant \mathbf{e}, \mathbf{d}} \mathcal{H}_{\mathbf{f}}$ of the locally closed subsets

$$
\mathcal{H}_{\mathbf{f}}=\left\{(\varphi, X) \in \mathcal{H} ; \operatorname{rk} \varphi_{i}=f_{i}, i \in Q_{0}\right\},
$$

and $\mathcal{L}_{\mathbf{f}}$ is the image of $\mathcal{H}_{\mathbf{f}}$ under the regular map sending $(\varphi, X)$ to $\left(\left(\operatorname{im} \varphi_{i}\right), X\right)$. Observe that $\mathcal{X}_{U}$ is the union $\bigcup_{0 \neq \mathbf{f} \leqslant \mathbf{d}, \mathbf{e}} \pi_{2}\left(\mathcal{F}_{\mathbf{f}}\right)$ of the images under the second projection, and thus

$$
\operatorname{dim} \mathcal{X}_{U} \leqslant \max _{0 \neq \mathbf{f} \leqslant \mathbf{d}, \mathbf{e}} \operatorname{dim} \mathcal{F}_{\mathbf{f}}
$$

Now consider the first projection $\pi_{1}: \mathcal{F}_{\mathbf{f}} \rightarrow \mathcal{L}_{\mathbf{f}}$. For $V \in \mathcal{L}_{\mathbf{f}}$, we have $\pi_{1}^{-1}(V)=\{V\} \times \mathcal{A}_{V}$, so we know by Corollary 3.2 that

$$
\operatorname{dim} \pi_{1}^{-1}(V) \leqslant \operatorname{dim} \operatorname{rep}(Q, \mathbf{d})-1-\lambda,
$$

as $V \subseteq U$ is a subrepresentation of $\tau T$ with $\operatorname{dim} V \leqslant \mathbf{d}$. We conclude that

$$
\begin{aligned}
\operatorname{dim} \mathcal{X}_{U} & \leqslant \max _{0 \neq \mathbf{f} \leqslant \mathbf{d}, \mathbf{e}} \operatorname{dim} \mathcal{F}_{\mathbf{f}} \leqslant\left(\max _{0 \neq \mathbf{f} \leqslant \mathbf{d}, \mathbf{e}} \operatorname{dim} \mathcal{L}_{\mathbf{f}}\right)+\operatorname{dim} \operatorname{rep}(Q, \mathbf{d})-1-\lambda \\
& \leqslant \eta(\mathbf{e})-1-\lambda+\operatorname{dim} \operatorname{rep}(Q, \mathbf{d}),
\end{aligned}
$$

which implies our claim. 
COROLlaRY 3.4. - Let $c=\max \left\{\eta\left(\tau T_{i}\right) ; i=1, \ldots, r\right\}$. Then the set

$$
\mathcal{E}_{\mathbf{d}}=\left\{X \in \operatorname{rep}(Q, \mathbf{d}) ;{ }^{1}[T, X]>0\right\}
$$

is either empty or else $\operatorname{codim} \mathcal{E}_{\mathbf{d}} \geqslant 1+\lambda-c$.

Proof. - If $T$ is projective then the set $\mathcal{E}_{\mathbf{d}}$ is empty. Otherwise, any non-zero map in

$$
\operatorname{Hom}_{Q}(X, \tau T) \simeq \operatorname{Ext}_{Q}^{1}(T, X)
$$

induces a non-zero map $X \rightarrow \tau T_{i}$ for some non-projective $T_{i}$, and we see that

$$
\mathcal{E}_{\mathbf{d}}=\bigcup_{T_{i} \text { non-projective }} \mathcal{X}_{\tau T_{i}} .
$$

The claim follows from Lemma 3.3.

Remark 3.5. - For $\lambda \geqslant c+n-r$, we have that either $\mathcal{E}_{\mathbf{d}}$ is empty or that $\operatorname{codim} \mathcal{E}_{\mathbf{d}} \geqslant 1+n-r$.

Now we concentrate on the set $\mathcal{Z}_{\mathbf{d}}^{\prime}=\left\{X \in \mathcal{Z}_{Q, \mathbf{d}} ;{ }^{1}[T, X]=0\right\}$.

LEMmA 3.6. - For $X \in \mathcal{Z}_{\mathbf{d}}^{\prime}$, there exists an epimorphism $X \rightarrow S=\bigoplus S_{j}$, where the sum is taken over the $n-r$ simple objects of $T^{\perp}$.

Proof. - We choose a basis $\left\{f_{1}, \ldots, f_{s}\right\}$ of $\operatorname{Hom}_{Q}(T, X)$ and we put

$$
f=\left(f_{1}, \ldots, f_{s}\right): T^{s} \rightarrow X
$$

Then any homomorphism from $T$ to $X$ factors through $f$. Let $X^{\prime}=\operatorname{im} f$ and $\bar{X}=\operatorname{coker} f$. The exact sequence

$$
0 \rightarrow X^{\prime} \rightarrow X \rightarrow \bar{X} \rightarrow 0
$$

induces the following long exact sequence

$$
\begin{aligned}
0 & \rightarrow \operatorname{Hom}_{Q}\left(T, X^{\prime}\right) \stackrel{g}{\rightarrow} \operatorname{Hom}_{Q}(T, X) \rightarrow \operatorname{Hom}_{Q}(T, \bar{X}) \\
& \rightarrow \operatorname{Ext}_{Q}^{1}\left(T, X^{\prime}\right) \rightarrow \operatorname{Ext}_{Q}^{1}(T, X) \rightarrow \operatorname{Ext}_{Q}^{1}(T, \bar{X}) \rightarrow 0 .
\end{aligned}
$$

Since there is an epimorphism $T^{\prime} \rightarrow X^{\prime}$ and since ${ }^{1}\left[T, T^{\prime}\right]=0$, we have ${ }^{1}\left[T, X^{\prime}\right]=0$. Moreover, $g$ is bijective by the universality of $f$ and, together with our assumption ${ }^{1}[T, X]=0$, this implies that $\bar{X} \in T^{\perp}$.

Recall that $[X, Y] \neq 0$ for all non-zero $Y \in T^{\perp}$ as $X$ lies in $\mathcal{Z}_{Q, \mathbf{d}}$. In particular, $\left[X, S_{j}\right] \neq 0$ for $j=1, \ldots, n-r$. Mapping the sequence (3.1) to $S_{j}$ and using that $\left[X^{\prime}, S_{j}\right] \leqslant\left[T^{\prime}, S_{j}\right]=0$, we find that $\left[\bar{X}, S_{j}\right] \neq 0$ for all $j$. But any non-zero morphism $\bar{X} \rightarrow S_{j}$ is surjective, because $\bar{X} \in T^{\perp}$ and $S_{j} \in T^{\perp}$ is simple. We obtain the required epimorphism by composing the projection $X \rightarrow \bar{X}$ with a surjective map $\bar{X} \rightarrow S=\bigoplus_{j=1}^{n-r} S_{j}$.

Since the set $\mathcal{Z}_{Q, \mathbf{d}}$ is given by $(n-r)$ equations, each irreducible component of $\mathcal{Z}_{Q, \mathbf{d}}$ has codimension at most $(n-r)$. Thus Theorem 1.1 follows from Remark 3.5 and the following fact.

PROPOSITION 3.7. - If $\mathcal{Z}_{\mathbf{d}}^{\prime}$ is not empty, then it is irreducible and $\operatorname{codim} \mathcal{Z}_{\mathbf{d}}^{\prime}=n-r$. 
Proof. - If $\mathcal{Z}_{\mathbf{d}}^{\prime}$ is non-empty, Lemma 3.6 tells us that $\mathbf{d} \geqslant \operatorname{dim} S$ and that $\mathcal{Z}_{\mathbf{d}}^{\prime}$ lies in

$$
\mathcal{A}_{S}=\{X \in \operatorname{rep}(Q, \mathbf{d}) ; \exists \text { epimorphism } X \rightarrow S\} .
$$

By Lemma 3.1, $\mathcal{A}_{S}$ is irreducible, and

$$
\operatorname{codim} \mathcal{A}_{S} \geqslant n-r-\left\langle\mathbf{d}, \mathbf{d}^{\prime \prime}\right\rangle=n-r-[T, S]+{ }^{1}[T, S]=n-r .
$$

As ${ }^{1}[T, X]=0$ is an open condition, $\mathcal{Z}_{\mathbf{d}}^{\prime}$ is open in $\mathcal{Z}_{Q, \mathbf{d}}$, and therefore codim $\mathcal{Z}_{\mathbf{d}}^{\prime} \leqslant n-r$. Thus $\mathcal{Z}_{\mathrm{d}}^{\prime}$ is open and dense in $\mathcal{A}_{S}$ and consequently irreducible.

\title{
Acknowledgements
}

The second author gratefully acknowledges support from the Polish Scientific Grant KBN No. 5 PO3A 00821 and Foundation for Polish Science. He also thanks the Swiss Science Foundation, which gave him the opportunity to spend a year at the University of Berne.

\section{REFERENCES}

[1] Chang C., Weyman J., Representations of quivers with free module of covariants, preprint.

[2] Gabriel P., Représentations indécomposables, in: Sém. Bourbaki (1973/74), exp. n. 444, in: Lecture Notes in Math., vol. 431, 1975, pp. 143-169.

[3] Kraft H., Geometrische Methoden in der Invariantentheorie, Vieweg Verlag, 1984.

[4] Littelmann P., Koreguläre und äquidimensionale Darstellungen, J. Algebra 123 (1989) 193-222.

[5] Popov V.L., Representations with a free module of covariants, Funct. Anal. Appl. 10 (1977) 242-244.

[6] Ringel C.M., The rational invariants of tame quivers, Invent. Math. 58 (1980) 217-239.

[7] RINGel C.M., Tame Algebras and Integral Quadratic Forms, in: Lecture Notes in Math., vol. 1099, Springer Verlag, 1984.

[8] Sato M., KimuRA T., A classification of irreducible prehomogeneous vector spaces and their relative invariants, Nagoya J. Math. 65 (1977) 1-155.

[9] SCHOFIELD A., Semi-invariants of quivers, J. London Math. Soc. 43 (1991) 385-395.

[10] SCHWARZ G.W., Representations of simple Lie groups with regular ring of invariants, Invent. Math. 49 (1978) 167-191.

[11] SchWARZ G.W., Representations of simple Lie groups with a free module of covariants, Invent. Math. 50 (1978) 1-12.

[12] Schwarz G.W., Lifting smooth homotopies of orbit spaces, Inst. Hautes Études Sci. Publ. Math. 51 (1980) 37-135.

\author{
Christine RiEDTMANN \\ Mathematisches Institut, Universität Bern, \\ Sidlerstrasse 5, \\ CH-3012 Bern, Switzerland \\ E-mail: christine.riedtmann@math-stat.unibe.ch
}

\author{
Grzegorz ZWARA \\ Faculty of Mathematics and Computer Science, \\ Nicholas Copernicus University, Chopina 12/18, \\ 87-100 Toruń, Poland \\ E-mail: gzwara@mat.uni.torun.pl
}

